\title{
Effect of Varying Magnetic Fields on Targeted Gene Delivery of Nucleic Acid- Based Molecules
}

\author{
Taha ÇIKIM ${ }^{1}$, Özlem ORAL ${ }^{2}$, Özlem ÜNAL ${ }^{3}$, Devrim GÖZÜAÇIK ${ }^{4}$, \\ Havva F. YAĞCI ACAR ${ }^{3}$, Ali KOŞAR ${ }^{1, *}$ \\ * Corresponding author: Tel.: +90 (216) 48396 21; Fax: +90 (216) 48395 50; \\ Email: kosara@sabanciuniv.edu \\ 1 Mechatronics Engineering Program, Sabanci University, TR \\ 2 Nanotechnology and Research Center, Sabanci University, TR \\ 3 Department of Chemistry, Koç University, TR \\ 4 Molecular Biology Genetics and Bioengineering Program, Sabanci University, TR
}

\begin{abstract}
The importance of high transfection efficiency has been emphasized in many studies investigating methods to improve gene delivery. Accordingly, non-viral transfection agents are widely used as transfection vectors to condense oligonucleotides, DNA, RNA, siRNA, deliver into the cell, and release the cargo. Polyethyleneimine (PEI) is one of the most popular non-viral transfection agents. However, the challenge between high transfection efficiency and toxicity of the polymers is not totally resolved. The delivery of necessary drugs and genes for patients and their transport under safe conditions require carefully designed and controlled delivery systems and constitute a critical stage of patients' treatment. Compact systems are considered as the strongest candidate for the preparation and delivery of drugs and genes under leak free and safe conditions because of their low energy consumption, low waste disposal, parallel and fast processing capabilities, removal of human factor, high mixing capabilities, enhanced safety, and low amount of reagents. Motivated by this need in the literature, a platform for gene delivery via magnetic actuation of nanoparticles was developed in this study. The use of PEI-SPION (Super paramagnetic ironoxide nanoparticles) as transfection agents in in vitro studies was investigated with the effect of varying magnetic fields provided by a special magnetic system design, which was used as magnetic actuator offering different magnet's turn speeds and directions in the system. Results obtained from magnetic actuator systems were compared to the experiments without actuation and significant enhancement was observed in the transfection efficacies.
\end{abstract}

Keywords: Ferrofluid, PEI, SPION, transfection, magnetic actuation, MCF-7, PC-3, DU-145, RT-4

\section{Introduction}

Magnetofection is a basic and very efficient transfection technique which uses magnetic fields to concentrate nucleic acidcontaining-nanoparticles into target cells. Classical transfection methods, including cationic lipids, polymers, electroporation and gene gun, were used with some limitations such as low efficiency and toxicity. Additionally, slow vector accumulation and consequently low vector concentration at target tissues were identified as strong barriers to effective viral or non-viral gene delivery [Luo D, Saltzman WM. Enhancement of transfection by physical concentration of DNA at the cell surface]. In terms of overcoming these inconveniences, magnetofection provides an alternative way of targeted gene delivery using magnetic fields. As a result, many studies related to gene delivery via magnetofection are now available in the literature [1-11]. For example, Scherer et al. [1] coupled gene vectors with superparamagnetic nanoparticles and targeted gene delivery by application of magnetic field to accomplish the difficulties of viral or non-viral gene therapy. They succeed to decrease duration of gene delivery to minutes and low retroviral titer was compensated. Moreover, they observed a high transfection efficiency in 
vitro, which was reproduced in vivo using magnetic field-guided local transfection of the gastrointestinal tract and blood vessels. Another in vivo study was performed by Kopke et al. [2]. They made middle ear implant capable to produce biomechanically relevant forces and provide auditory function by using superparamagnetic iron oxide nanoparticles (SNPs). For this aim, they covered oleic acid and dextran coated magnetic SNPs with silica and polymer materials in order to generate adequate forces for pulling nanoparticles across the tissue of in vivo models. Subramanian et al. [3] revealed increased transfection efficiency in both human prenatal cardiac progenitor cells and adult cardiomyocytes by oscillating magnet array-based nanomagnetic exposure. In an experimental and simulation demonstration, Lin et al. [4] showed in vitro site-specific gene transfection and enhanced efficiency by an electric field-assisted electroporation microchip. In this study, they revealed that plasmid DNA could be delivered at desired regions of cells using an electrostatic force. According to magnetofection principle, Huang et al. [5] developed an innovative electroporation (EP) system, which has an EP microchip combined with electrophoresis (ES). They used a lab-on-a-chip system to manipulate adherent cells in situ without detachment of the EP-ES microchip and showed significant increase in gene transfection efficiency by applying attracting electromagnetic field. Fung et al. [6] investigated cellular internalization efficiency of electrodeposited ferromagnetic nickel nanowires into $3 \mathrm{~T} 3$ fibroblast cells in magnetic field, and showed the absence of inflammatory responses following cellular incubation with these nanowires. Namiki et al. [7] showed a new nanoparticle formulation composed of oleic acid-coated magnetic nanocrystal core and a cationic lipid shell, which can be magnetically guided to deliver genes more efficiently in cells and tumors. Stride et al. [8] investigated the effect of both ultrasonically and magnetically-mediated DNA transfection of Chinese hamster ovary $(\mathrm{CHO})$ cells. They compared in vitro uptake of DNA by cells exposed to ultrasound and/or a magnetic field in the presence of phospholipid coated microbubbles prepared with or without a suspension of magnetic nanoparticles. The results indicated that the transfection efficiency was significantly enhanced due to simultaneous exposure of cells to ultrasound and magnetic field. Kim et al. [9] reported on the interfacing of whole cells with biocompatible lithographically defined ferromagnetic microdiscs with a spin-vortex ground state. It was found that spin-vortexmediated stimulus caused compromised integrity of cellular membranes and initiation of programmed cell death. Moreover, the authors succeed $90 \%$ cancer-cell destruction in vitro by applying low-frequency magnetic field of few tens of hertz.

In recent years, there has been much interest in using siRNAs and nanoparticles for silencing genes for the gene therapy of selected diseases. To achieve successful gene silencing using siRNA, the molecule must survive in the extracellular environment before reaching the cell surface, cross the cell membrane and then enter RISC complex where unwinding and pairing of the antisense strand occur with native mRNA. Lee et al. [10] covalently linked magnetic nanoparticles to siRNAs, which targeted cyclic Arg-Gly-Asp (RGD) peptides together with fluorescent dyes to make stable simultaneous delivery and multimodal imaging. Veiseh et al. [11] reported the development of biocompatible, magnetic nanovectors for effective intracellular delivery of siRNA. They synthesized magnetic nanoparticles coated with either pArg or PEI and functionalized with siRNA, and showed pArg-coated nanovectors most effectively knocked-down target genes.

Gene therapy is a promising and developing therapeutic method that has the potential to be used in the treatment of various diseases, including cancer. Development of safe and efficient gene delivery systems is still a major challenge for the success of gene therapy. Here, we designed a specific magnetic actuator, which include fundamental features such as magnet's turn speed and magnetic 
field direction control. For gene delivery via magnetic actuation, we used super paramagnetic ironoxide nanoparticles (SPION) coupled with polyethyleneimine (PEI) and assessed DNA uptake efficacy in several cell lines derived from different tissues, namely MCF-7 (breast), RT-4 (urinary bladder), PC-3 and DU-145 (prostate) cells.

\section{Experimental Setup and Procedure}

Moving a magnetic field without distorting its shape is trivial if the source of magnetic field can be moved in the same direction. It was decided that such a mechanism would be cumbersome at best. Instead, the linear movement of the magnet was mimicked with magnets rotating around a common axis. The idea behind this design is using the synchronous rotation of symmetric and opposing magnets to generate a magnetic field, which peaks when the magnets are at the closest position and which diminishes while the magnets are at the farthest position. Rare earth magnets with $300 \mathrm{mT}$ magnetic field strength are placed on to the each face of the square profile rotors with the (i-1) $9 \mathrm{a} \mathrm{mm}$ distance from the reference edge, where $i$ is the magnet number and $a$ is the magnet diameter. The rotors are placed in such a way that the tube, which has $3 \mathrm{~mm}$ outer diameter, stays in between the rotors, which are actuated by a simple DC motor. Angular velocities are calculated from the position data, which are obtained from the encoder of the motor. The first design has been improved by reducing the angular separation of rare earth magnet pairs to obtain higher flow rates, fewer discontinuities on the magnetic field, and better position tracking. This was achieved by changing rectangular rotors into hexagonal rotors.

In this experimental investigation, a specific magnetic actuator was designed in order to accomplish targeted gene delivery of nucleic acid-based molecules and it included fundamental features such as magnet's turn speed and magnetic field direction control. The figure of magnetic actuator is illustrated in
Fig. 1. The system consists of two magnetic rotors, DC motor $(12 \mathrm{~V})$, power switch, speed control switch, power cables, miniature gears and proper stand and units for placing cell dish.

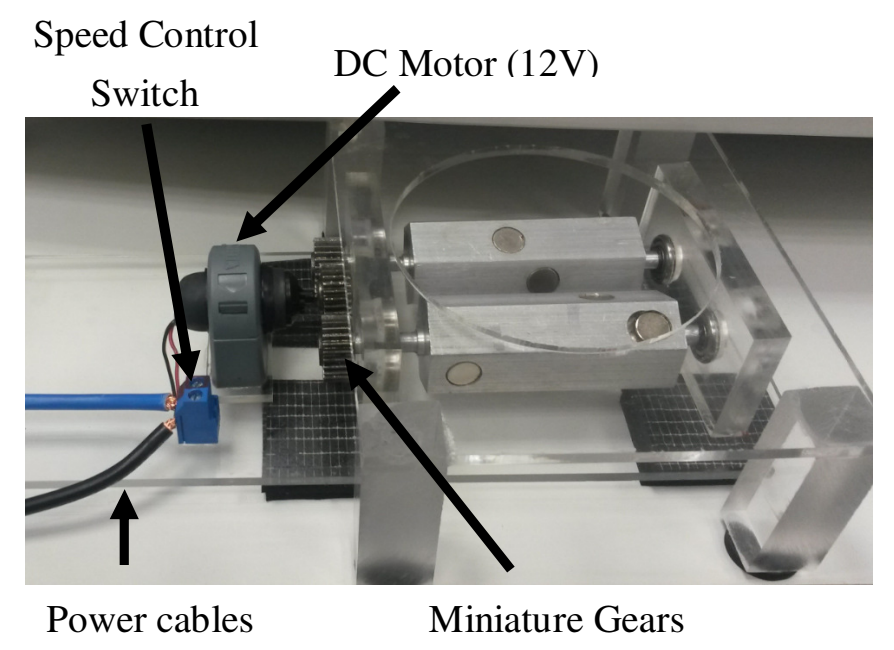

Figure 1: Schematics of magnetic actuator

The most important parameter of magnetic field requires significantly better control, enhanced accuracy to achieve optimal magnetic conditions for gene delivery. Therefore, the magnetic actuator was designed in order to provide improved control ability, durability and less system complexity. Thanks to miniature gears and speed control switch, turn speed of magnetic rotors can be adjustable to the desired values. Hence, it provides to have control ability of magnetic field force by changing rotor speed. On the other hand, the direction of magnetic field plays an essential role. In order to supply continuous magnetic field, each magnets on the magnetic rotors are transversely placed. Hence the system provides magnetic field in both vertical and horizontal directions due to magnetic vortexes which is shown in Fig. 2. 


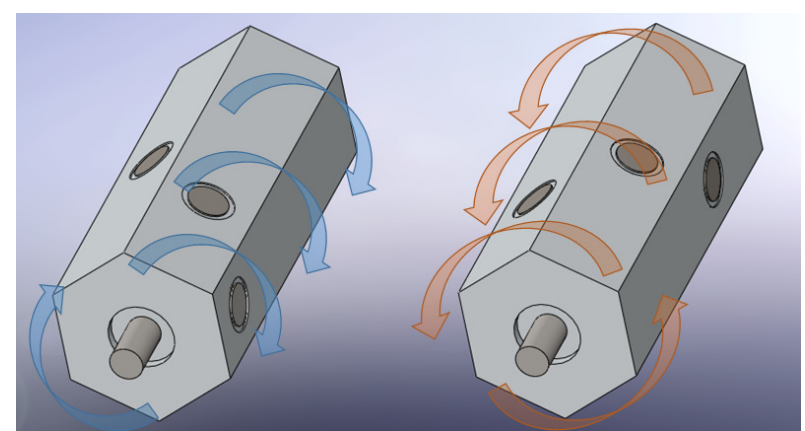

Figure 2: Magnetic field direction on magnetic rotors

\subsection{Preparation of polyethyleneimine- superparamagnetic nanoparticles (PEI- SPION)}

Polyethyleneimine-superparamagnetic nanoparticles (PEI-SPION) were kindly provided from Koç University, Department of Chemistry, Turkey. In brief, oleic acid coated $\mathrm{Fe}_{3} \mathrm{O}_{4}$ nanoparticles were obtained from the Ocean NanoTech LLC. The hydrophobic $\mathrm{Fe}_{3} \mathrm{O}_{4}$ nanoparticles were silica coated by a modified reverse micro emulsion procedure by using tetraethyl orthosilicate. Polyethyleneimine (PEI) was added to the surface of the $\mathrm{Fe}_{3} \mathrm{O}_{4}-\mathrm{SiO}_{2}$ nanoparticles by stirring with trimethoxysilylpropyl modified polyethyleneimine (50\% in isopropanol).

\subsection{Plasmid DNA isolation}

Plasmid DNA isolation was performed with plasmid DNA purification kit according to manufacturers instructions (Nucleobond Xtra Midi/Maxi, Macherey-Nagel, Germany). pMAX-GFP (Addgene, Cambridge, USA) plasmid was used for transfection tests.

\subsection{Cell Culture}

MCF-7, human breast cancer cell line, PC-3 and DU-145, human prostate cancer cells were cultured in DMEM (Sigma, Germany) supplemented with 10\% FBS (Biochrom KG, Germany), $\quad 1 \% \quad$ Penicillin-Streptomycin (Biological Industries, Israel), 1\% L-glutamine (Biological Industries, Israel). RT-4, urinary bladder cancer cells were cultured in McCoy's (Biochrom KG, Germany) complete medium. $1,5 \times 106$ cells were seeded on $10-\mathrm{cm}$ culture plate in $10 \mathrm{ml}$ cell culture medium. Cells were incubated at $37^{\circ} \mathrm{C}$ in a $5 \% \mathrm{CO} 2$ atmosphere.

\subsection{DNA Transfection and Microscopic Analyses}

According to the cell type $15-40 \mu \mathrm{g}$ of PEI or PEI-SPION was added into eppendorf tube containing $100 \mu \mathrm{l}$ DMEM or McCoy's (without serum and antibiotics). In another eppendorf tube, $5 \mu \mathrm{g}$ of pMAX-GFP was mixed with $100 \mu$ l DMEM or McCoy's (without serum and antibiotics). Contents of the tubes were mixed and vortexed for 15 seconds. Following $10 \mathrm{~min}$. incubation at room temperature, the mixture was added dropwise onto $10 \mathrm{~cm}$ culture plates. Cells were washed with PBS after 6-8 hours and incubation was continued in DMEM or McCoy's supplemented with $10 \%$ FBS, $1 \%$ PenicillinStreptomycin and 1\% L-glutamine. At $48 \mathrm{~h}$ post-transfection, cells were stained by addition of $1 \mu \mathrm{g} / \mu \mathrm{l}$ Hoechst 33342 (Life Technologies, USA) and further incubation for $30 \mathrm{~min}$., and then analyzed under a fluorescent microscope (Olympus BX60).

\section{Results and Discussion}

\subsection{PEI Transfection Results}

PEI is one of the most widely and most successfully used polymers used for nucleic acid delivery. However, high transfection efficiencies might be associated with toxicity. To assess gene delivery efficiency of SPIONcoupled-PEI via magnetic actuation, we first performed some control experiments with naked PEI. We transfected MCF-7, PC-3, DU145 and RT-4 cells, with different ratios of GFP-tagged DNA and PEI molecules in order to obtain maximum transfection efficiency with minimal toxicity. Maximum transfection efficiency was obtained using $2.5 \mu \mathrm{g}$ DNA with $20 \mu \mathrm{g}$ PEI for PC-3 and DU-145 cells. For MCF-7 and RT-4 cells, best transfection ratios were achieved using $35 \mu \mathrm{g}$ and $15 \mu \mathrm{g}$ PEI, respectively. (Fig.3 left two panel and Fig.4). As seen under phase-contrast microscope images, under our experimental conditions, we did not observe any significant toxicity. Yet, usage of PEI amounts more than $35 \mu \mathrm{g}$ caused dramatic cell death in four different cancer cell lines. 


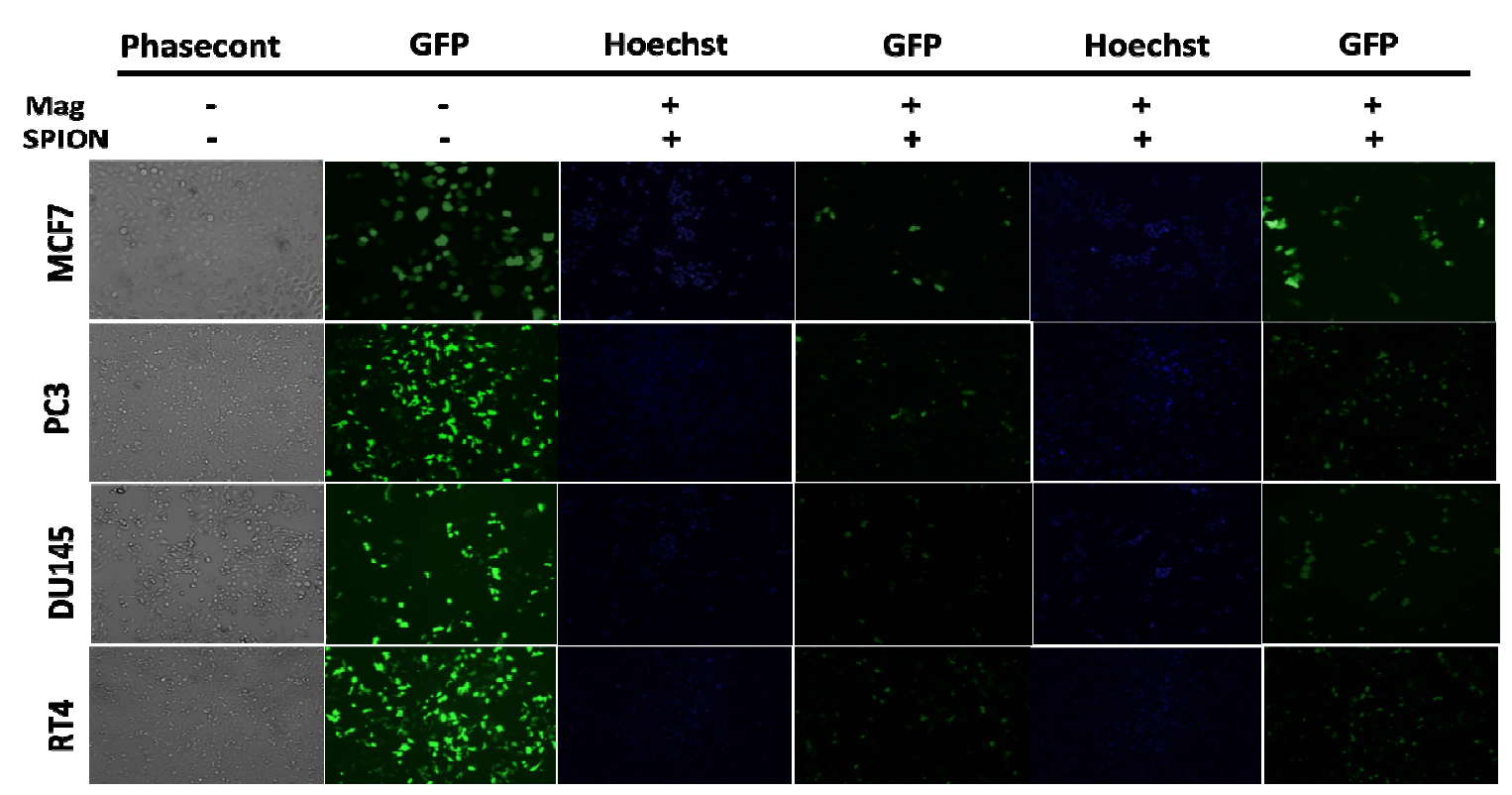

Figure 3: PEI transfection with or without SPION under magnetic or non-magnetic conditions in MCF-7, PC-3, DU-145 and RT-4 cell lines. $2.5 \mu \mathrm{g} / \mu 1$ GFP-tagged DNA was used in combination with $20 \mu \mathrm{g}$ PEI for PC-3 and DU-145, 35 $\mu \mathrm{g}$ for MCF-7, $15 \mu \mathrm{g}$ for RT-4 cells (Left two panel). For PEI-SPION transfection, $6 \mu \mathrm{g}$ PEI-SPION/5 $\mu \mathrm{g}$ DNA was used under magnetic and nonmagnetic conditions. $48 \mathrm{~h}$ after transfection, cell images was taken by using inverted fluorescent microscope

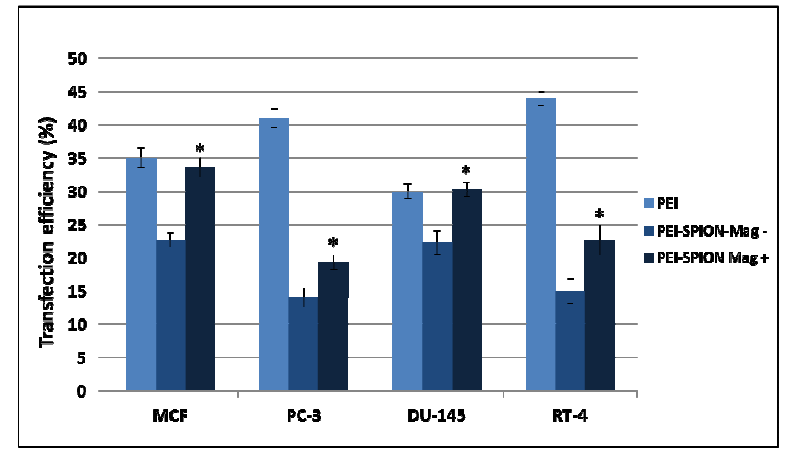

Figure 4: PEI/PEI-SPION transfection efficiency under magnetic or non-magnetic conditions in MCF-7, PC-3, DU-145 and RT-4 cell lines. Data are shown as mean \pm S.D. (n $=3), \quad(* \mathrm{p}<0.05)$

In order to investigate effect of varying magnetic fields on targeted gene delivery of nucleic acid-based molecules, experiments are conducted using the miniature magnetic actuation device under various test conditions. Accordingly, the experimental tests are performed with different magnetic rotor turn velocities varying from 0.108 to $0.216 \mathrm{~m} / \mathrm{s}$ when the supplied voltage was $12 \mathrm{~V}$ and currents were $2.1 \mathrm{Amp}$ and $4.2 \mathrm{Amp}$, respectively. In order to assess the relationship between magnetic rotor velocity and supplied power, calculated voltage/current curve is given in Fig. 5.

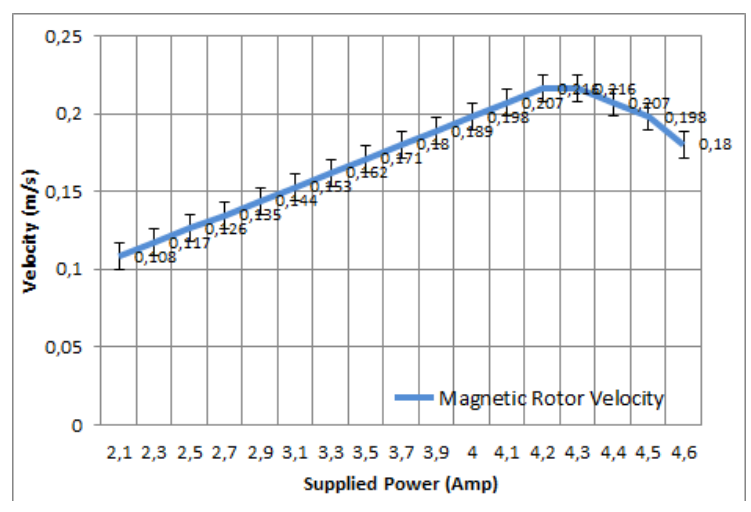

Figure 5: Power vs velocity curve for magnetic rotors 


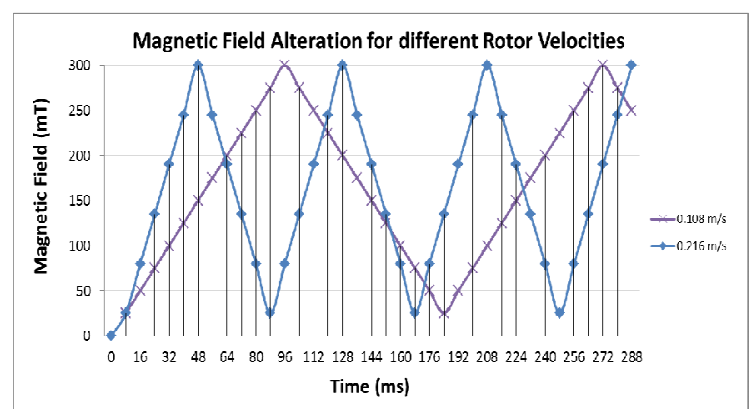

Figure 6: Magnetic field alteration for different rotor velocities

The use of PEI-SPION (Super paramagnetic iron oxide nanoparticles) as transfection agents was investigated with the effect of varying magnetic fields provided by a special magnetic system design, which was used as magnetic actuator offering different magnet's turn speeds in the system. PEI complexes bind to DNA via the electrostatic binding interaction between secondary amine groups of PEI and phosphate backbone of DNA. On the other hand, PEI complexes bind to DNA via the electrostatic binding interaction between secondary amine groups of PEI and phosphate backbone of DNA [12]. In the relevant literature [13], it was showed that silica layer over iron oxide core can easily bind to amine groups of PEI with amine-to-silica interaction. In order to assess magnetic field effect, we first performed a concentration test for PEISPION and the concentration, which gives maximum transfection efficiency $(6 \mu \mathrm{g}$ PEISPION/5 $\mu$ g DNA) without any toxicity is used for further experiments. All cancer cells exposed with or without magnetic field for 6 hours then washed with PBS and continue to culture for additional 42 hours. Results were obtained via miniature magnetic actuation device. Significant enhancements for targeted gene delivery were attained for all cell types when compared with non-magnetic field conditions. (Fig. 3 last four panel and Fig. 4). However, when we compared with single PEI, PEI-SPION has much lower transfection efficiency. The results indicated that, PEISPION can be successfully used for gene delivery in MCF-7, PC-3, DU-145 and RT-4 cell lines.
In previous studies, PEG (Polyethyleneglycol)-PEI-SPION was used for transfection agent in SGC-7901 (Human Gastric Carcinoma) cell line [35]. This work showed that PEGylated-PEI-SPION can be perfect candidate for gene delivery of nucleic acid based molecules as being transfection agents in in vitro studies. In addition of this, here we showed PEI-SPION could also be a perfect candidate without any modification such as PEGylation. SPIONs could be concentrated at certain region such as tumors because of their magnetic nature. Beside, magnetic nanoparticles could be used as hyperthermia treatment. They cause local temperature increase which may also be used as an alternative and efficient cancer therapy. Therefore, increased gene delivery efficiency with magnetic field that we showed here could be turned to alternative treatment approach for cancer therapy.

\section{Conclusions}

In this study, a platform for gene delivery via magnetic actuation of nanoparticles was developed. PEI-SPION (Super paramagnetic iron oxide nanoparticles) was investigated as transfection agents in in vitro studies with the effect of varying magnetic fields offered by a miniature magnetic actuator design. The proposed device could be used as magnetic actuator, which offers different magnet's turn speed and directions. The major findings of this study are as follows:

- PEI-transfection was optimized without any toxicity effect in four different cancer cell lines (MCF-7, PC3, DU-145, RT-4) and transfection efficiency was obtained up to $45 \%$.

- PEI-SPION molecules can be successfully used for nucleic-acid based gene delivery and magnetic field exposure can improve the delivery efficiency.

- Higher magnet's turn speed result in higher transfection rate. 
- Transfection rate is independent from magnet's turn direction.

- It is proven that the miniature magnetic actuator setup could be used for investigation of transfection agents in in vitro and in vivo studies.

- It is proven that the miniature magnetic actuator could deliver drug and genes to the targeted cell having low energy consumption, low waste disposal, and parallel and fast processing capacity, high mixing capability, enhanced safety and low amount of reagents.

\section{References}

[1] Scherer, F., Anton, M., Schillinger, U., Henke, J., Bergemann, C., Krüger, A., Gansbacher, B., and Plank, C., 2002, "Magnetofection: Enhancing and Targeting Gene Delivery by Magnetic Force in vitro and in vivo", Gene Therapy, 9 (2), pp. 102109.

[2] Kopke, R. D., Wassel, R. A., Mondalek, F., Grady, B., Chen, K., Liu, J., Gibson, D., and Dormer, K. J., 2006, "Magnetic Nanoparticles: Inner Ear Targeted Molecule Delivery and Middle Ear Implant”, Audiology and Neurotology, 11 (2), pp. $123-133$.

[3] Subramanian, M., Lim, J., and Dobson, J., 2013, "Enhanced Nanomagnetic Gene Transfection of Human Prenatal Cardiac Progenitor Cells and Adult Cardiomyocytes", Plos One, 8(7).

[4] Lin, Y., Li, M., and Wu, C., 2004, "Simulation and Experimental Demonstration of the Electric Field Assisted Electroporation Microchip for in vitro Gene Delivery Enhancement", Lab on a Chip, 4 (2), pp. $104-108$.

[5] Huang, K. S., Lin, Y. C., Su, C. C., and Fang, C. S, 2006, "Enhancement of an Electroporation System for Gene Delivery Using Electrophoresis with a Planar Electrode", Lab on a Chip, 7 (1), pp. $86-92$.

[6] Fung, A. O., Kapadia, V., Pierstorff, E., Ho, D., and Chen, Y., 2008, "Induction of Cell Death by Magnetic Actuation of Nickel Nanowires Internalized by Fibroblasts", The Journal of Physical Chemistry C, 112 (39), pp. 15085 -
15088.

[7] Namiki, Y., Namiki, T., Yoshida, H., Ishii, Y., Tsubota, A., Koido, S., Nariai, K., Mitsunaga, M., Yanagisawa, S., Kashiwagi, H., Mabashi, Y., Yumoto, Y., Hoshina, S., Fujise K., and Tada, N., 2009, "A Novel Magnetic Crystal-lipid Nanostructure for Magnetically Guided in vivo gene Delivery", Nature Nanotechnology, 4, pp. $598-606$.

[8] Stride, E., Porter, C., Prieto, A. G., and Pankhurst, Q., 2009, "Enhancement of Microbubble Mediated Gene Delivery by Simultaneous Exposure to Ultrasonic and Magnetic Fields", Ultrasound in Medicine \& Biology, 35 (5), pp. $861-868$.

[9] Kim, D. H., Rozhkova, E. A., Ulasov, I. V., Bader, S. D., Rajh, T., Lesniak, M. S., and Novosad, V., 2009, "Biofunctionalized Magneticvortex Microdiscs for Targeted Cancer-cell Destruction”, Nature Materials, 9 (2), pp. 165-171.

[10] Lee, H., Lee, K., Moon, S. H., Lee, Y., Park, T. G., and Cheon, J., 2009, "All-in-One TargetCell-Specific Magnetic Nanoparticles for Simultaneous Molecular Imaging and siRNA Delivery", Angewandte Chemie International Edition, 48 (23), pp. 4174 - 4179.

[11] Veiseh, O., Kievit, F. M., Mok, H., Ayesh, J., Clark, C., Fang, C., Leung, M., Arami, H., Park, J. O., and Zhang, M., 2011, "Cell Transcytosing poly-arginine Coated Magnetic Nanovector for Safe and Effective siRNA Delivery", Biomaterials, 32 (24), pp. $5717-5725$.

[12] Mc, Bain, S.C., Yiu, H.P., El, Haj, A., Dobson, J., 2007, "Polyethylenimine Functionalized Iron Oxide Nanoparticles as Agents for DNA Delivery and Transfection", Material Chemistry, 17, pp. 2561-2565.

[13] Jang, E.S., Park, K.S., 2012, "Lipofectamine2000 Assisted Magnetofection to Fibroblast Cells Using Polyethyleneimine-Fe3O4-SiO2 Nanoparticles", Bull. Korean Chem. Society, 8 